\title{
ESTRATEGIAS RETÓRICAS EN LA CONSTRUCCIÓN DEL PERSONAJE JUDÍO DEL DIALOGUS CONTRA IUDAEOS DE PEDRO ALFONSO
}

\section{Rhetorical devices in the construction of the Jewish character of Peter Alfonsi's Dialogus contra iudaeos}

\section{Estratégias retóricas na construção do caráter judeu do Dialogus contra iudaeos de Pedro Alfonso}

\author{
Dra. Natalia G. Jakubecki \\ Jefa de trabajos prácticos de Historia de la Filosofía Medieval \\ Facultad de Filosofía y Letras - Universidad de Buenos Aires, \\ Investigadora Asistente (CONICET), \\ ORCID: https://orcid.org/0000-0003-1674-7064 \\ E-mail: jakubecki@gmail.com
}

Recebido em: 10/08/2020

Aprovado em: 08/12/2020

\begin{abstract}
Resumen: El objetivo de este trabajo es dar cuenta de algunas estrategias retóricas en la configuración identitaria de Moisés, el personaje judío del Dialogus contra iudaeos, escrito por Pedro Alfonso de Huesca $c$. 1106. Ello en vista de arribar a una conclusión que permita ponderar la presencia o ausencia de ciertos elementos textuales de la literatura antijudía posterior.

Palabras clave: Identidad - Retórica - Pedro Alfonso de Huesca.

Abstract: The present paper aims to expose the different rhetorical strategies in the identity configuration of Moses, the Jewish character on the Dialogus contra Iudaeos, written by Peter Alfonsi of Huesca $c$. 1106. This first exposition has a subsequent purpose: to evaluate the presence or absence of some textual components into the following anti-Jewish literature. Keywords: Identity - Rhetoric - Peter Alfonsi of Huesca.
\end{abstract}




\section{Introducción}

Un hito importante en la historia literaria del antijudaísmo ha sido, sin lugar a dudas, el texto de Pedro Alfonso de Huesca conocido como el Dialogus contra iudaeos (o los Dialogi), cuya fecha de composición se supone alrededor de 1106 . No ha de sorprender que resultara fuente de inspiración para encarnizados defensores del cristianismo de la talla de Raimundo Martí o Alonso de la Espina desde el momento en que, de la serie de diálogos controversiales escritos en el occidente latino medieval, es el primero cuyo propósito explícito consiste en refutar algunas creencias judías e, incluso, musulmanas. Propósito que, por otra parte, excedía -y en mucho- las empresas más bien pedagógicas de sus congéneres, en especial las precedentes. De allí que este conjunto de doce diálogos se convirtiera en el escrito antijudío de consulta casi obligada en los siglos inmediatamente posteriores. Los 79 testimonia de él que nos han llegado son buena prueba de ello. ${ }^{1}$

Mi intención en lo que sigue, pues, es dar cuenta de la configuración identitaria del personaje judío, y ello en vista de una conclusión que permita ponderar la presencia o ausencia de ciertos elementos textuales de la literatura antijudía posterior. ${ }^{2}$

Para cumplir cabalmente con este objetivo, el presente artículo se dividirá en tres momentos. El primero consistirá en una presentación general del autor y de la obra. El segundo atenderá a la forma específica del texto y a la función que el personaje judío cumple en él. En el tercero, finalmente, se analizará la construcción identitaria de dicho personaje a partir de la recuperación de los diversos dispositivos textuales en los que se esta se apoya, con particular atención a aquellos de índole retórica.

\section{El autor y la obra ${ }^{3}$}

Moisés Sefardí (a. 1086-c. 1125) fue un destacado intelectual de la comunidad judía, docto en medicina, física, filosofía y, especialmente, astronomía. Según él mismo narra en el prefacio de su Dialogus, cambió su nombre por el de Pedro Alfonso el día en que fue bautizado por la fe cristiana (Dial., prólogo: 198). ${ }^{4}$ Ese acontecimiento es el 
único del cual poseemos la fecha exacta (29 de junio de 1106) y, por tanto, término de referencia para las restantes dataciones biográficas. ${ }^{5}$

Según las declaraciones del propio Pedro, su conversión había suscitado suficiente malestar entre sus antiguos correligionarios como para que le fueran dirigidas serias amonestaciones:

\begin{abstract}
“[Algunos decían] que había aceptado la ley y la fe de los cristianos [...] porque hasta tal punto me había despojado de toda modestia que despreciaba a Dios y su ley. Otros, en cambio, decían que yo lo había hecho porque no había interpretado bien las palabras de los profetas y de la ley. Pero otros lo achacaban a vanagloria y, calumniándome, decían que lo había hecho buscando la gloria del mundo porque había visto que el pueblo de los cristianos superaba a todos los demás" (Dial., prólogo: 199).
\end{abstract}

Fueron estas increpaciones las que, como cuenta, lo empujaron a escribir el texto que nos ocupa. Sin embargo, Pedro no se conforma con hacer una apología de sí mismo ni de la religión cristiana. Como hemos anticipado, tiene como objetivo, además, "la destrucción de las creencias de todas las otras gentes" (omnium aliarum gentium credulitas destructionem) (Dial., prólogo: 199). John Tolan ha notado con acierto que Pedro no sólo se dispone a probar que el cristianismo es compatible con la verdad y la razón, sino también que únicamente el cristianismo y ninguna otra religión lo es (1996a: XXII).

En este sentido, el Dialogus puede leerse como "el primer ejemplo de una nueva actitud", pero no solo porque se valga de las propias fuentes judías para volverlas en contra del judaísmo, como sostuvo Amparo Alba Cecilia (2005: 30), sino también porque el mismo propósito refleja de algún modo la forma mentis escolástica que estaba ya dando sus primeros pero firmes pasos en aquél entonces. En efecto, una buena argumentación ya no se limitará a dar pruebas en favor de la propia tesis sino que, además, será necesario refutar su contraria. Ello nos permite entender que el autor tiene, en realidad, un único propósito, solo que dividido en dos momentos, los cuales se reflejan en la estructura de la obra, consecuentemente dividida en dos partes: una pars destruens conformada por cuatro libros dedicados a refutar las creencias del judaísmo y uno solo en contra de las del Islam, y una pars construens que a lo largo de siete libros explica las tesis conflictivas pero, según Pedro, "verdaderas", de la fe cristiana. 
Resta señalar que nuestro autor comenta que eligió este formato por cuestiones didácticas, o sea, "para que el ánimo del lector tuviera mayor facilidad para entender" (Dial., prólogo: 199). En ello es consecuente con las tendencias literarias de su época, para la cual el diálogo se convierte en un modelo estilístico "particularmente apto para la enseñanza de las artes y de la religión” (VON Moos, 1989: 994). Tal es así que, hacia finales del siglo XI y principios del XII, el subgénero de diálogos adversos iudaeos -si es que se lo puede catalogar como tal- no solo aumenta su volumen, sino que comienza a desarrollar nuevas estrategias textuales, tanto respecto del contenido teórico como de orden retórico. El Dialogus de Pedro Alfonso, pues, será pionero en más de un aspecto (cf. RESNICK, 2009: 85-86).

\section{Forma del texto y función de Moisés}

De todos estos aspectos, uno de los más interesantes y el que, según creo, hace a esta obra sumamente peculiar (mucho más aún que el hecho de que su autor fuera un judío converso) es que los dos interlocutores que Pedro Alfonso elije para desarrollar el diálogo son él mismo, sólo que escindido diacrónicamente. Esto es, la voz antagonista del cristianismo se encuentra representada por el personaje de Moisés, cuyo nombre e identidad religiosa coinciden con las del autor antes de su bautismo, mientras que la defensa del cristianismo y el ataque a las otras creencias estarán a cargo de Petrus, alter ego del autor ya convertido. ${ }^{6}$ Sin embargo, para que la trama fuera verosímil, en la ficción narrativa el personaje de Petrus presenta al de Moisés como un amigo de la infancia que, apenas se entera de su conversión, acude a visitarlo, alarmado, para pedirle que disputen "en el campo de la razón" (uterque in alterne rationis campo discurramus) (Dial., I: 201).

Como señala Thomas Ricklin, si bien este desdoblamiento al mejor estilo "Dr. Jekyll y Mr. Hyde" es usual en la literatura latina medieval (los Soliloquia de Agustín, la Consolatio de Boecio y los Synonyma de Isidoro de Sevilla son claros ejemplos de "diálogos autocontemplativos" [selbstbetrachtende Dialogs]), en el caso de personajes judíos resulta un fenómeno aislado. Observa el autor que no deja de ser insólito incluso dentro de la literatura latina misma, en la medida en que el desdoblamiento del ego se 
da por medio del momento del bautismo cristiano, es decir, de un renacimiento en otra identidad religiosa (1999:147-149).

De hecho, en lo que concierne específicamente a los diálogos controversiales, este también se muestra excepcional. A diferencia de todos ellos, nuestro Dialogus pareciera ser un soliloquio, género que se encuentra en el límite entre diálogo y monólogo. En efecto, el soliloquio, por una parte, consiste en un monólogo interior con un yo escindido en el que el conflicto se da en el seno de la identidad pero, por otra parte, aun cuando su interlocutor sea el sí mismo, se trata de un sí mismo que se transforma por este diálogo interior. Sin embargo, en el Dialogus de Pedro Alfonso la escisión del yo propia de los soliloquios no se da a partir de algún conflicto interno que necesite disiparse. Se trata más bien de un recurso retórico que el autor eligió para reforzar literariamente su nueva identidad. Y, más aún, eligió hacerlo contrastándola con la otredad; pero no con una cualquiera sino con aquella a la que él perteneció, y de la que, en definitiva, proviene la que ha adoptado recientemente. Puede incluso agregarse que esta conformación de la propia identidad recientemente asumida se hace teniendo en mente a la nueva comunidad de pertenencia ante la cual debía justificarse, el cristianismo. En efecto, la mayoría de los estudios dedicados a esta obra coinciden en que, de haber querido abrir un diálogo real con sus antiguos correligionarios, como el mismo autor arguye en el prefacio, Pedro podría haber escrito el diálogo en hebreo, lengua con la que estaba familiarizado. Pero no solo prefirió hacerlo en latín sino que, además, decidió comenzar su texto con una profesión de fe que poco y nada podría interesarle a un lector judío (cf. v.g., RiCKIN, 1999: 152-154; PALMEN, 2016: 545-546).

Es cierto que Pedro Alfonso parte de su propia experiencia, pero Petrus y Moisés no ofician de voces autobiográficas -a pesar del breve panegírico de este último hacia Petrus en el inicio del diálogo (Dial., I: 201)- sino como una construcción ficcional deliberada que integra elementos de dos comunidades religiosas cuyos discursos el autor conoce muy bien. De hecho, este diálogo es el primero en su género que incluye literatura rabínica y ofrece la traducción latina de algunos pasajes del Talmud, ${ }^{7}$ lo cual asegura la efectiva inclusión de los principios doctrinales de ambos interlocutores en la conformación de sus respectivas identidades religiosas. A diferencia de otros diálogos en los que mientras que los autores cristianos poseen una comprensión parcial y 
mediada de las creencias judías, Pedro logra replicar un discurso más auténtico por la simple razón de que lo conocía de primera mano.

Con todo, si bien la voz del judaísmo es parte constitutiva del diálogo, lo es en gran medida no por las intervenciones de Moisés, sino porque Petrus la retoma con el único fin de objetarla. La contribución de Moisés al intercambio no es tanto la de aportar argumentos en favor de su propia fe, sino la de ser -como bien dice José González Luis- un "cómodo opositor" en la medida en que no complica demasiado a Petrus con sus preguntas (1992: 256), aunque, a decir verdad, la mayor parte de los personajes judíos hasta el momento tampoco ponían en aprietos a sus interlocutores cristianos. $^{8}$

Dejando a un lado el quinto diálogo, que versa sobre el Islam y en el que Moisés interviene alternativamente como portavoz de los sarracenos y como un oyente curioso, nuestro personaje judío cumple diversas funciones en tanto antagonista: realiza preguntas de naturaleza exegética más o menos complejas, propone él mismo ciertos temas que le interesa tratar, acepta algunas explicaciones pero exige que profundicen otras e, incluso, llega a señalar una contradicción en la exposición de Petrus -que éste, como es esperable, resuelve sin ninguna dificultad (cf. Dial., IV: 289). Lo único que no hace Moisés es ser él mismo el portavoz de sus propios argumentos.

Y esto nos conduce al tercer y último momento de este trabajo, la construcción identitaria de Moisés.

\section{Construcción identitaria de Moisés}

Los especialistas comparten la opinión de que Pedro Alfonso construye el personaje judío más verosímil de la literatura controversial escrita hasta entonces y ello se debe, desde luego, a su propia biografía. Con todo, no porque el autor provenga de la comunidad judía quiere decir que su personaje judío no se trate de una construcción que, como tal, es interesada, subjetiva y ficcional. Interesada, en tanto que Pedro pretende que el personaje cristiano, Petrus, pueda "ganarle" a Moisés -si se me permite la expresión- esto es, destruir sus creencias y demostrar la superioridad de las propias. Para ello, Moisés tiene que mostrarse falible e ignorar ciertas cosas de antemano. 
Subjetiva, pues tal construcción se halla erigida únicamente sobre la percepción que el autor tiene de sí y de sus ex correligionarios, en la que no intervienen ni percepciones ni voces ajenas que contribuyan a conformar la identidad de modo colectivo. En consecuencia, en tanto construcción particular, es también necesariamente sesgada. Y ficcional, porque no intenta ofrecer una descripción lo más ajustada posible a lo que él considera que es la realidad, sino que elabora un personaje ficticio con elementos reales. De lo contrario, Moisés no sería "un amigo de la infancia". Más aún, si se perdiera de vista que no solo Moisés sino también Petrus son ficcionales, aun cuando discrepen respecto a sus convicciones de fe, deberían compartir los mismos conocimientos científicos pues uno y otro serían la misma persona, lo cual, a su vez, haría que los largos pasajes dedicados a explicaciones geográficas, cosmogónicas, metafísicas, etc. fueran del todo innecesarios.

Demás está decir que ello no es así. Petrus, en tanto personaje, cuenta con una erudición muchísimo más amplia que la de su interlocutor judío quien, por su parte, es introducido en escena por el cristiano del siguiente modo:

\footnotetext{
"Cuando le llegó la noticia de que yo, abandonada la ley paterna, había elegido la fe cristiana, vino desde su lugar de residencia y se presentó rápidamente ante mí con semblante de hombre indignado. Increpándome, me saludó no con expresiones del viejo amigo, sino [...] como si fuera un extraño" (Dial., I: 200-201).
}

Al acudir ofendido a la casa de Petrus para pedir explicaciones, Moisés nos es presentado como alguien movido ante todo por la emoción, alguien que "coloca su propio modelo de vida como norma incuestionable y condena sin previo juicio e irreflexivamente" (GONZALEZ LuIS, 1992: 259). Pero ello es solo en apariencia porque, en realidad, tras el primer impulso mantiene la compostura y cordialidad. Y, lo que es más importante aún, este "perfectissimus amicus" (Dial., prólogo: 8) de Petrus es a tal punto sensato, que él mismo es quien propone una disputa racional de modo que pueda juzgar si la conversión del flamante cristiano fue o no justa (Dial., I: 201). Pero a pesar de que una de las reglas propuestas por el judío y aceptadas de mutuo acuerdo consiste en que ambos puedan preguntar, responder y oponerse a la explicación del otro (Dial., I: 202), Moisés "no está en situación de rebatir a Petrus", como señala Cardelle de Hartmann (2011:1049). 
La relación asimétrica que ambos personajes mantienen con el saber se muestra en el desconcierto por momentos explícito de Moisés. Tan solo a modo de ejemplo, tomemos el primer diálogo, en el que tras fallidos intentos por defenderse de la imputación de que los judíos suscriben al antropomorfismo divino, ${ }^{9}$ nuestro personaje admite carecer de otra explicación, a lo cual Petrus le responde "Gracias a Dios, ya no me das una respuesta necia" (iam paulisper stulticiae cares) y un poco más adelante, agrega, "Ya que vuestra argumentación busca refugiarse en tan irracional (irrationabilis) conclusión, apoyándote en las tradiciones de los antiguos te será posible afirmar cualquier mentira" (Dial., I: 207). Frente a tan mordaces intervenciones, la réplica de Moisés no es precisamente la de un sabio, ni siquiera la de un par. Es la de alguien desconcertado: "He respondido no por razón de certeza (non ratione recti) sino buscando hallar un modo de salida" (Dial., I: 208). Solo tras cinco pedidos de explicación nuestro personaje está en condiciones de decir "Por fin (tandem) lo entiendo bien" (Dial., I: 211).

Por otra parte, es posible hallar algunas intervenciones de Moisés en las que este expresa su agradecimiento a Petrus por instruirlo. En la primera parte del debate, aquella en la cual Petrus se detiene en los errores exegéticos del judaísmo, luego de que le explicara a Moisés que es imposible que Dios llore porque ello implicaría un cambio en una naturaleza cuya inmutabilidad nadie pone en cuestión, Moisés confiesa: "Ya que sin interrupción soy hecho más docto por ti (per te doctor fio), también continuamente te doy las gracias" (Dial., I: 219). Un pasaje similar se encuentra en el quinto diálogo, en el que Pedro Alfonso le deja a Moisés un inusitado espacio para expresarse y, aun así, es Petrus quien termina teniendo razón y Moisés reconociéndolo: "Bien me corregiste y, dando gracias a Dios, aprendí con tus palabras la prudencia” (Dial., v: 301).

Lo que me interesa señalar con esto es que si bien es cierto que el que debe dar explicaciones es Petrus, este tipo de intervenciones, aunque infrecuentes, tienden a acentuar la pasividad de Moisés. Ritva Palmén acierta en ver este recurso una estrategia retórica que termina por convertirlo en una especie de colega o, más aún, de estudiante cristiano que solo de tanto en tanto cuestiona las opiniones de un Petrus (2016: 550) quien, en contrapartida, deviene maestro. ${ }^{10}$ Sin embargo, esta actitud de Moisés no es gradual, como sostiene la autora. Por el contrario, a medida que el diálogo avanza y Petrus refuta una y otra vez las opiniones de Moisés, el judío deja a un lado la gratitud 
inicial y permite entrever cierto fastidio. Tal es el caso de un pasaje en el que ambos se encuentran debatiendo acerca de los ritos pascuales:

\begin{abstract}
"M.- Una vez que me has desautorizado la festividad de la Pascua, que entre todas es la más sobresaliente y notable y de la que se dice que quien no la celebra perecerá, no vale la pena que hablemos de otras de menor importancia, ya que mucho más fácilmente podrías quitarles trascendencia" (Dial., XII: 391).
\end{abstract}

La asimetría entre un personaje y otro rebasa los límites epistémicos y se hace manifiesta también en el trato que, si bien amable, no es en absoluto de igual a igual. Compartimos la observación de Tolan cuando afirma que "a pesar de su tono sereno y educado" los Dialogi exponen "una condena áspera y absolutamente nueva del judaísmo" (1996b: 181). Condena que no pasa por agravios directos a Moisés -en definitiva los dos "Stultissime omnium" (IX: 146 y X: 158) que le dirige Petrus hablan más de su falta de paciencia que se la capacidad intelectual de su interlocutor- sino por la severidad con que refuta sus creencias. ${ }^{11}$ De hecho, no hace falta remitirnos a estudios contemporáneos para detectarlo. Basta atender al mismo personaje de Moisés, que advierte que las palabras de Petrus "son breves y leves pero en ellas hay intención dura y grave" (Dial., I: 204).

Pese a ello, nuestro judío se encuentra más motivado en aprender de su oponente que en discutirle, en preguntar más que en contestar. Ese es, después de todo, el papel que le toca en tanto personaje judío nacido de la pluma de un autor cristiano, por muy recién convertido que fuera -o quizá justamente a causa de ello. Un papel no menor si se toma en cuenta que, como hemos dicho, Pedro Alfonso se sirve de Moisés para construir su propia identidad, una en la que el peso de la razón es crucial (cf. FUNKENSTEIN, 1971: 378). De allí que el nivel de inteligencia propia que quiera ostentar ha de ser medianamente proporcional al de su interlocutor, pues de lo contrario: o bien debería esgrimir argumentos sencillos y superficiales -lo cual no es el caso- para que Moisés los entendiera, o bien sería él mismo un necio al malgastar su tiempo desarrollando sutilezas que el judío es incapaz de comprender. Por tanto, el hecho de que Moisés pueda seguir las intrincadas demostraciones de Petrus lo convierte ipso facto sino en un par, al menos en alguien con un nivel intelectual tal que, a diferencia de 
"las mentes simples de los menos doctos [que] no perciben las sutilezas de las cosas profundas" (Dial., III: 275-276), es capaz de apreciar verdades racionales e, incluso, la misma "superioridad de Pedro", como nota Palmén (2016: 555). Y ello a pesar de que ocasionalmente pida que se profundice en tal o cual aspecto del argumento lo cual, por otra parte, no deja de ser un recurso retórico del propio autor, por lo general, una excusa para discurrir sobre cuestiones aledañas y menores que desea exponer pero que no hacen al argumento principal.

Con todo, ello no implica que Moisés sea sumiso ni dócil. "Bien me has enredado con tus insidias (Bene michi insidiatus)", le achaca a Petrus ya cerca del final (Dial., X: 362). Pero el que hayan desacuerdos y que el intercambio tenga, incluso, sus momentos álgidos no es más que un requerimiento de verosimilitud (cf. v.g. Dial., II: 264-265; VII: 318-19; IX: 350-351). Lo que sí considero que resulta relevante es el hecho de que no una sino dos veces Moisés acepte la racionalidad de los argumentos de su interlocutor y, aun así, se rehúse a convertirse al cristianismo (cf. Dial., V: 291; XII: 399). Y ello lo sabemos no porque lo diga de manera explícita, sino porque, de lo contrario, no habría motivos para que Petrus invocara la iluminación del Espíritu Santo:

\footnotetext{
"M.- Es cierto que Dios te dio mucha de su sabiduría y te dotó de gran razón, cosas a las que yo no puedo vencer; al contrario, eres tú quien refutaste mis objeciones con tus razonamientos.

P.- [...] Y su tú también creyeras lo que nosotros creemos y te hicieras bautizar tendrías esa misma iluminación del Espíritu Santo para conocer lo verdadero y rechazar lo falso" (Dial., XII: 399-400).
}

Lo que está haciendo Moisés es negarse a aceptar como verdadero todo un conjunto de proposiciones de fe que se reconoce incapaz de refutar. Si nos atenemos al enfoque racionalista que predomina en el pensamiento de Pedro Alfonso en general, y en esta obra en particular, y reparamos en las reiteradas ocasiones en las que ambos personajes aluden a la irracionalidad de las creencias de judías, ${ }^{12}$ este final, de manera implícita, acusa a Moisés de insensato e intelectualmente inferior. ${ }^{13}$

Ahora bien, advirtamos que fue Moisés mismo quien propuso disputar en el campo de la razón, y que, a su vez, reconoce que esa razón quedó innegablemente del lado de su oponente cristiano. Ricklin ve en este final un gesto positivo por parte del 
autor, según el cual estaría indicando que aunque se reconozca la inferioridad argumentativa de la comprensión rabínica de las Escrituras y la lógica asista de modo unilateral al cristianismo, los judíos no tienen por qué verse forzados a convertirse (199: 154). Esa es una lectura posible. Sin embargo, me permito proponer otra que, de todas formas, no es necesariamente incompatible. Creo que por mucho que a nivel ficcional Petrus exprese la esperanza de que, como le sucedió a él, su amigo se convierta, ya solo por este final y sin contar los muchos otros elementos teóricos y retóricos que se nos presentan a lo largo del texto, Pedro Alfonso se inscribe, por desgracia, en la larga lista de autores cristianos que acusan a los judíos de pertinaces, puesto que ven la verdad y no la siguen.

En este punto se me podrá decir, y con justa razón, que es necesario distinguir entre la construcción identitaria del personaje y la que se hace de la comunidad judía, y solo después analizar si ambas se condicen -y así Moisés sería un mero portavoz de ella- ${ }^{14}$ o no se condicen en absoluto -lo cual lo convertiría en una excepción carente de toda representatividad. No obstante, esta es una tarea de más largo aliento que reservamos para un trabajo posterior.

\section{Conclusiones}

Para concluir al menos este tramo del estudio, me permito una breve reflexión. He dicho al comenzar que los Dialogi de Pedro Alfonso fueron de consulta casi obligada para los polemistas antijudíos inmediatamente posteriores. Pero mientras esta obra -a semejanza de gran parte de las disputas ficcionales de finales del siglo XI y comienzos del XII- se esfuerza por mostrar mutuo respeto entre los dialogantes y en matizar los rasgos identitarios de Moisés de modo que sea un digno interlocutor, aquellas que le siguieron exhibieron un trato cada vez más agresivo y una construcción de los personajes judíos que, en términos generales, los mostraba como pertinaces, mentirosos y/o irracionales. Tales son, pues, las adjetivaciones tanto explícitas como implícitas que en ellas predominan.

Además -y de manera un tanto paradójica, si se quiere- con cuanto mayor fidelidad y cuidado se recogía el discurso ajeno, con mayor virulencia se lo atacaba (cf. SOUSSEN, 2013). Ello coincide con el momento en el que los diálogos literarios dejaban atrás su 
función de pedagógica intracristiana y comenzaban a utilizarse como un modelo para polemizar con un "otro" real en la vida cotidiana. ${ }^{15}$

Con todo, resulta significativo que a medida que la judeofobia se iba afianzando en los círculos letrados de la Europa cristiana, el formato de diálogo fuera cayendo en desuso y su lugar, ocupado por tratados y manuales de índole inquisitorial (cf. DAHAN, 2006: 4179). En este sentido, no podemos dejar de mencionar el caso del Speculum Historiale, pues no solo es paradigmático, sino que se vincula directamente con nuestra fuente. Allí, Vicente de Beauvais, su autor, reprodujo los primeros cinco libros del Dialogus de Pedro Alfonso (es decir, la pars destruens) pero con ligeros arreglos que le permitieron prescindir de la figura de Moisés convirtiendo, de ese modo, el diálogo original en un tratado condenatorio. Este gesto, esta decisión literaria, nos lleva a concluir que aun cuando el texto de Pedro Alfonso tenga un fuerte componente antijudío, el hecho mismo de estar escrito con el formato diálogo no es poca cosa. En primer lugar, porque incluso si no escapa por completo del sesgo de la subjetividad del autor, es mucho más plural que un tratado en tanto ofrece a la voz ajena un espacio objetivo (cf. FIDORA, 2009: 81). Y en segundo lugar, porque a pesar de que se trate de una ficción literaria, la elección de este formato es una muestra -aunque más no sea indeliberada- de que el autor consideraba a un judío como un sujeto de diálogo. Es cierto que al traer a la discusión textos menores o interpretaciones de estos a los que la misma comunidad judía poco o ningún caso hacía (como en el primero de los diálogos, a propósito del antropomorfismo de Dios), ${ }^{16}$ Pedro Alfonso parece querer minar la credibilidad del oponente para poner en duda, incluso, su idoneidad como tal. Con todo, haya sido pensado o no sinécdoque de su comunidad, Moisés no deja de ser, en efecto, un interlocutor posible dentro del conjunto de interlocutores válidos.

\section{Bibliografía}

\section{Fuentes}


PEDRO ALFONSO DE HUESCA. Diálogo contra los judíos. LACARRA, Ma Jesús (coord.); TOLAN, John (intr.); MiETH, Klaus-Peter (texto latino) y DUCAY, Esperanza (trad.). Huesca: Instituto de estudios altoaragonenses, 1996.

PETRUS VENERABILIS. Adversus iudeorum inveteratam duritiem. En: FrIEDMAN, Yvonne (ed.). Corpus Christianorum Continuatio Mediaevalis (CCCM 58). Turnhout: Brepols, 1985.

\section{Complementaria}

ALBA CECILIA, Amparo. "Pedro Alfonso versus Moisés Sefardí". En: IZQUIERdo BENITO, Ricardo y MORENO KoCH, Yolanda (coords.). Del pasado judío en los reinos medievales hispánicos: afinidad y distanciamiento: XIII Curso de Cultura Hispanojudía y Sefardí de la Universidad de Castilla-La Mancha. Cuenca: Universidad de Castilla-La Mancha 2005.

CARDELLE DE HARTMANN, Carmen. "Diálogo literario y polémica religiosa en la Edad Media (900-1400)”. En: GonZALEZ MuÑoz, Fernando, GonZalez, Antonio y Macias Villalobos, Cristóbal (eds.). Actas del Congreso Internacional “Cristianismo y tradición latina. Madrid: Ediciones del Laberinto, 2001, pp. 103-123.

CARDELLE DE HARTMANN, Carmen. 'Pedro Alfonso y su 'Dialogus': estado de la cuestión”. En: Martinez GazQuez, José, De la Cruz Palma, Oscar y Ferrero HERNANDEZ, Cándida (eds.). Estudios del latín medieval hispánico. Florencia: SISMEL Edizioni del Galluzzo, 2011, pp. 1049-1057.

DAHAN, Gilbert. The Christian Polemic against the Jews in the Middle Ages. Notre Dame: University of Notre Dame Press, 1998 [reimp. 2006].

FIDORA, Alexander. "Das philosophische Religionsgespräch im Mittelalter: von Gilbert Crispin und Peter Abaelard zu Ramon Llull’. En: SPRINGER, Bernd y FIDORA, Alexander (eds.). Religiöse Toleranz im Spiegel der Literatur. Eine Idee und ihre ästhetische Gestaltung. Münster: Lit-Verlag, 2009. pp. 71-81. 
FUNKENSTEIN, Amós. "Basic Types of Christian Anti-Jewish Polemics in the Later Middle Ages". Viator 2 (1971), pp. 373-382.

GONZÁLEZ LUIS, José. “El Dialogus de Pedro Alfonso”. Fortunatae 4 (1992), pp. $245-262$

JAKUBECKI, N. “Construcción y función de la figura del 'gentil' en dos diálogos medievales. Los personajes de Gilberto Crispino y del Pseudo-Anselmo". Ideas y valores 67/168 (dic. 2018), pp. 267-287.

MARTÍNEZ GÁZQUEZ, José. "El lenguaje de la violencia en el prólogo de la traducción latina del Corán impulsada por Pedro el Venerable”. CEHM 28 (2005), pp. 243-252.

PALMÉN, Ritva. “Agreement in Conflict: Peter Alfonsi’s Dialogi contra Iudaeos and the Idea of Recognition". Medieval Encounters 22 (2016), pp. 540-564.

RESNICK, Irven. "Introduction”. En: Idem. Petrus Alfonsi, Dialogue against the Jews, Washington DC: The Catholic University of America Press, 2006.

RESNICK, Irven. "La portée historique du Dialogue contre les juifs de Petrus Alfonsi”. Les cahiers du judaïsme 25, (2009), pp. 83-101.

RICKLIN, Thomas. "Der Dialogus des Petrus Alfonsi. Eine Annäherung”. En: JACOBI, Klaus (ed.). Gespräche lesen. Philosophische Dialoge im Mittelalter. Tubinga: Gunter Narr, 1999, pp. 139-156.

SOUSSEN, Claire. "La parole de l'autre, a prise en compte des arguments de l'adversaire dans la polémique anti-juive à la fin du Moyen Âge". En: MorLET, Sébastien, MunNich, Olivier y POUDERON, Bernard (eds.). Les dialogues adversus iudaeos. Permanences et mutations d'une tradition polémique. París: Institut d'Études Augustiniennes, 2013, pp. 351-367.

TOLAN, John. Petrus Alfonsi and His Medieval Readers. Florida: University Florida Press, 1993.

TOLAN, John. "Introducción”. En: LACARRA, Mª Jesús (coord.). Pedro Alfonso de Huesca. Diálogo contra los judíos. Huesca: Instituto de Estudios Altoaragonenses, 1996a, pp. IX-LX. 
TOLAN, John. “Los Diálogos contra los judíos”. En: LACARRA, Mª Jesús (coord.). Estudios sobre Pedro Alfonso de Huesca. Huesca: Instituto de Estudios Altoaragoneses, 1996b, pp. 181-226.

VON MOOS, P. “Le dialogue latin au Moyen Âge: l'exemple d'Evrard d'Ypres”. Annales. Économies, Sociétés, Civilisations 44, n. 4 (1989), pp. 993-1028.

\section{Notas}

\footnotetext{
${ }^{1}$ La lista completa, junto a sus respectivas descripciones, se encuentra en TOLAN, 1993: 182-198. Para la recepción medieval del Dialogus a la que hemos hecho referencia, puede consultarse IDEM: 95-131, y también RESNICK 2006: 27-36.

${ }^{2}$ Un valioso aporte para la confección de este artículo ha sido el trabajo de Ritva PALMÉN (2016) en el que la autora releva algunos componentes de la praxis retórica positiva que opera en la obra. Sin embargo, mientras ella tiene por objetivo aplicar las nociones de la "Teoría del reconocimiento" (Theory of Recognition) como instrumento hermenéutico, mi intención en concentrarme en las estrategias utilizadas en la confirmación de la identidad de un personaje en particular.

${ }^{3}$ En este apartado nos ceñiremos a traer a la memoria del lector o lectora algunos contenidos que deberá tener presentes para seguir la exposición, pero no es nuestra intención realizar una presentación exhaustiva. Para ello, puede consultarse, de entre la abundante bibliografía al respecto, TOLAN, $1996 \mathrm{a}$.

${ }^{4}$ Raimundo Martí, en su Pugio fidei, lib. II, dist. 3, c. 4, dice que Pedro Alfonso fue un "magnus rabinus apud judaeos". Sin embargo, esta atribución resulta dudosa. De ahora en adelante cito el texto de Pedro Alfonso de Huesca por ubicación interna (prólogo o número de diálogo) y paginación de la traducción al castellano de DUCAY: 1996.

${ }^{5}$ Sobre los interrogantes que aún quedan acerca de su vida y obra, véase CARDELlE DE HARTMANN, 2011.

${ }^{6}$ Como es usual en estos casos, dejo el nombre del personaje en su versión latina para no confundirlo con el del autor.

${ }^{7}$ En un artículo publicado en 2011, Cardelle de Hartmann señaló que si bien estos pasajes ya habían sido identificados, restaba aún establecer la rama de transmisión textual de la que proceden. Cf. CARDELLE DE HARTMANN, 2011: 1049, y n. 3.

${ }^{8}$ En aquella época, la excepción más evidente -y por ello una de las más interesantes en cuanto al lugar que ocupa en la discusión el personaje judío- es la Disputatio inter iudaei et christianum de Gilberto Crispino.

${ }^{9}$ Tal como señala Tolan: "En este punto Pedro Alfonso ridiculiza la tradición mística conocida como Shi' ur Qomah (medida del cuerpo) que suponía especular sobre las dimensiones del cuerpo de Dios, [...] objeto de enérgicos ataques por parte de los judíos caraítas e incluso muchos judíos rabbanitas albergaban ciertos recelos al respecto..." (1996a: XXV). Véase al respecto RESNICK, 2009: 89-93.

${ }^{10}$ Este es un rasgo bastante usual en los diálogos de la época. El más llamativo al respecto es la Disputatio inter christianum et gentilem del Pseudo-Anselmo, en el cual el personaje que comienza siendo gentil y aparece como tal al comienzo de cada una de sus intervenciones, termina siendo apuntado como "discipulus fidelis" hacia el último tercio del texto, mientras que su interlocutor pasa de ser "christianus" a "magister". Para un análisis detallado, véase JAKUBECKI, 2018.

${ }^{11}$ Las arriba consignadas, sumada al también referido pasaje "iam paulisper stultitiae cares", son las únicas ocasiones en las que Petrus insulta de manera personal y directa a Moisés, si es que no me he pasado por alto alguna (y aun si así lo fuera, no modifica el hecho de que sea un recurso inusual). La utilización de adjetivos o frases peyorativas dirigidos a las creencias y/o a la comunidad judía, en cambio, son mucho más frecuentes. Véase, solo a modo de ejemplo, la última intervención de Petrus del Dial., I: 46-50 (lat.); 242-246 (cast.). El diálogo IX, en el que se intenta demostrar que en Cristo se cumplen todas las profecías, también es particularmente incisivo.

${ }^{12} \mathrm{Si}$ bien esta serie de calificaciones recorre la obra completa, véanse especialmente los primeros cuatro diálogos.

${ }^{13}$ Quien terminará por radicalizar este enfoque será, tan solo unos años más tarde, Pedro el Venerable: “¿Pero qué debo hacer? Si comienzo a responder esta o cualquier otra locura similar, yo mismo pareceré
} 
loco $[. .$.$] ¿Acaso no parecerá un lunático aquel que se esfuerza por debatir razonablemente con el hombre$ en quien toda razón ha sido sepultada, y que no ofrece nada más que comentarios vanos y tontos?", en Petrus Venerabilis. Adversus iud.: 126, 59-65. Traducción propia. Un excelente trabajo directamente ligado a Pedro el Venerable y la retórica, aunque no sobre el judaísmo sino sobre el Islam, es el de Martínez Gázquez: 2005.

${ }^{14}$ Esta es, desde luego -y concordando con los especialistas-, mi hipótesis inicial. Véase aquí la nota 11. Con todo, considero que aún falta un estudio exhaustivo para despejar toda duda.

15 Existe abundante literatura al respecto. Recomiendo especialmente el trabajo de CARDELLE DE HARTMANN: 2001 ya que, además de analizar diferentes casos y proponer una convincente clasificación de diferentes tipos de diálogos de este subgénero, ofrece un nutrido (aunque no completo) repertorio de los mismos.

${ }^{16}$ Véase aquí la nota 9. 